\title{
Renormalizability and Newtonian potential in scale-invariant gravity
}

\author{
Yun Soo Myung ${ }^{a}$ \\ Institute of Basic Science and Department of Computer Simulation, \\ Inje University, Gimhae 50834, Korea
}

\begin{abstract}
$\underline{\text { Abstract }}$
There is a conjecture that renormalizable higher-derivative gravity has a finite classical potential at the origin. In this work we show clearly that the scaleinvariant gravity (SIG) satisfies the conjecture. This gravity produces the better-behaved $1 / k^{4} \mathrm{UV}$ behavior as needed for renormalizability. It turns out that the SIG has the linear classical potential of $V \propto r$ and it is a UV complete theory.
\end{abstract}

${ }^{a}$ ysmyung@inje.ac.kr 


\section{Introduction}

There was a conjecture that renormalizable higher-derivative gravity has a finite Newtonian potential at the origin [1]. This relation was first mentioned in Stelle's seminal work [2] which showed that the fourth-derivative gravity (FDG) is renormalizable and has a finite potential at origin. However, this gravity belongs to a nonunitary theory because it has a massive pole with negative residue which could be interpreted either as a state of negative norm or a state of negative energy. In this case, a massive ghost tensor and a massive healthy scalar contribute in such a manner to cancel out the Newtonian singularity originated from a massless tensor.

Recently, it was conjectured that the reverse of the above statement is not true [3. It implies that the finiteness of the Newtonian potential at the origin is a necessary but not sufficient condition for the renormalizability of the model. The models considered in [3, 4] are different versions of sixth-derivative gravities. One is a fully six-derivative gravity which satisfies the above conjecture, whereas the other is a half six-derivative gravity which does not satisfy the reverse of the statement. That is, the latter is the case that even though the potential is finite at $r=0$, it is known to be non-renormalizable by power counting. More recently, it was probed that the necessary condition for (super-)renormalizability implies the sufficient condition for the cancellation of the Newtonian singularities in $D \geq 4$-dimensions [5].

In this work, we will show clearly that the scale-invariant gravity (SIG) satisfies the above conjecture. This gravity is considered as a very suitable model for exploring the connection between classical potential and renormalizability of the theory. We note that this theory has the better-behaved $1 / k^{4}$ tensor and scalar propagators as needed for renormalizability. It turns out that the SIG provides the linear classical potential of $V \propto r$ and it is a UV complete theory.

\section{Scale-invariant gravity(SIG)}

A classically scale-invariant gravity in four dimensions is defined by

$$
S_{\mathrm{SIG}}=\int d^{4} x \sqrt{-g}\left[-\frac{1}{a}\left(\frac{1}{2} C_{\mu \nu \rho \sigma}^{2}+\frac{w}{3} R^{2}\right)+c G B\right]
$$

where $C_{\mu \nu \rho \sigma}$ is the Weyl tensor and $R$ is the Ricci scalar. Also $G B=$ $R_{\mu \nu \rho \sigma}^{2}-4 R_{\mu \nu}^{2}+R^{2}$ is the Gauss-Bonnet topological term expressed by the divergence of a current $B^{\mu}$ as $G B=\nabla_{\mu} B^{\mu}$. Hence it makes no contribution 
to the equations of motion and we may ignore it when formulating the Feynman rules for the quantum theory. In other words, $G B$ does not contribute to the classical potential. One notes that the effect of this term is not relevant for one-loop renormalization, but it may play a role in renormalization group equations. The three coupling constants of $a$ (Weyl coupling), $w$ (Ricci coupling), and $c$ (Gauss-Bonnet coupling) are dimensionless and hence it is easy to derive their renormalization group equations.

The action (11) without $G B$ was first introduced as a gravity model for admitting linearized solutions with negative energy but all exact solutions possess zero energy [6]. On later, this was considered continuously as a prototype for renormalization and renormalization group analyses [7, 8, 9, 10, 11]. Hence, the SIG is considered as a very suitable model for exploring the close connection between classical potential and renormalizability of the theory.

On the other hand, we note that if one includes the Einstein-Hilbert term $2 R / \kappa^{2}$ with $\kappa^{2}=4 \kappa_{4}\left(\kappa_{4}=8 \pi G\right)$, the corresponding action is not scaleinvariant and was employed to prove the renormalizability of the FDG [2]. Furthermore, ignoring $R^{2}$ leads to the conformal (Weyl) gravity with the Gauss-Bonnet term. This model was first considered in [7], whereas on later two of [12, 13] have checked and corrected [7].

At this stage, we introduce the well-known relation

$$
C_{\mu \nu \rho \sigma}^{2}=2 W+G B, W=\left(R_{\mu \nu}^{2}-\frac{1}{3} R^{2}\right)
$$

which implies $C^{2}=2 W$ up to total divergence. Using (2), (11) can be written as

$$
\tilde{S}_{\mathrm{SIG}}=\int d^{4} x \sqrt{-g}\left[-\frac{1}{a} R_{\mu \nu}^{2}+\frac{1}{3 a}(1-w) R^{2}+\left(c-\frac{1}{2 a}\right) G B\right],
$$

which is useful to derive the equation of motion and the propagator. In order to find the classical potential, one has first to obtain the propagator. For this purpose, we expand the metric tensor $g_{\mu \nu}=\eta_{\mu \nu}+h_{\mu \nu}$ around the Minkowski metric $\eta_{\mu \nu}=(+---)$. Bilinearizing the Lagrangian of Eq. (3) together with imposing the de Donder gauge, one obtains $\mathcal{L}_{\text {SIG }}^{\text {bil }}=h^{\mu \nu} \mathcal{O}_{\mu \nu, \alpha \beta} h^{\alpha \beta} / 2$ [4, 14] with

$$
\mathcal{O}_{\mu \nu, \alpha \beta}=-\frac{1}{2 a} \square^{2} P_{\mu \nu, \alpha \beta}^{(2)}-\frac{2 w}{a} \square^{2} P_{\mu \nu, \alpha \beta}^{(0-s)}+\cdots .
$$

Inverting $\mathcal{O}$, we obtain the propagator for the SIG in momentum space

$$
\mathcal{D}_{\mu \nu, \alpha \beta}^{\mathrm{SIG}}(k)=-\frac{2 a}{k^{4}} P^{(2)}-\frac{a}{2 w k^{4}} P^{(0-s)}+(\cdots) .
$$


Here $P^{(2)}$ and $P^{(0-s)}$ represent the Barnes-Rivers operators

$$
\begin{aligned}
P_{\mu \nu, \alpha \beta}^{(2)} & =\frac{1}{2}\left(\theta_{\mu \alpha} \theta_{\nu \beta}+\theta_{\mu \beta} \theta_{\nu \alpha}\right)-\frac{1}{3} \theta_{\mu \nu} \theta_{\alpha \beta}, \\
P_{\mu \nu, \alpha \beta}^{(0-s)} & =\frac{1}{3} \theta_{\mu \nu} \theta_{\alpha \beta}, \quad \theta_{\mu \nu}=\eta_{\mu \nu}-\frac{k_{\mu} k_{\nu}}{k^{2}},
\end{aligned}
$$

while $(\cdots)$ denotes the set of terms that are irrelevant to the spectrum of the theory. Note that (5) without $(\cdots)$ represents a gauge-invariant part of the propagator. Also it does not include $1 / k^{2}$ propagator appearing in the FDG. The propagator (5) carries 6 DOF: two massless tensors (4 DOF) + two massless scalars (2 DOF) since the former propagator $1 / k^{4}$ is the degenerate limit of the massless tensor pole and the massive ghost pole (a massless tensor dipole) as well as the later one is the degenerate limit of the massless scalar pole and the massive scalar pole (a massless dipole). The 6 DOF is compared to 8 DOF for the FDG.

By setting $k_{0}=0$, the spatial part of the gauge-invariant propagator (5) takes the form

$$
\begin{aligned}
\mathcal{P}_{\mu \nu, \kappa \lambda}^{\mathrm{SIG}}(\mathbf{k})= & -\frac{2 a}{\mathbf{k}^{4}}\left[\frac{1}{2}\left(\eta_{\mu \kappa} \eta_{\nu \lambda}+\eta_{\mu \lambda} \eta_{\nu \kappa}\right)-\frac{1}{3} \eta_{\mu \nu} \eta_{\kappa \lambda}\right] \\
& -\frac{a}{\mathbf{k}^{4}} \frac{\eta_{\mu \nu} \eta_{\kappa \lambda}}{6 w} .
\end{aligned}
$$

We note the relation between the classical potential sourced by a static mass $M$ and propagator

$$
V(r)=\frac{M}{4(2 \pi)^{3}} \int d^{3} \mathbf{k} e^{i \mathbf{k} \cdot \mathbf{r}} \mathcal{P}_{00,00}(\mathbf{k}) .
$$

Fourier-transforming

$$
\mathcal{P}_{00,00}^{\mathrm{SIG}}(\mathbf{k})=-\frac{4 a}{3} \frac{1}{\mathbf{k}^{4}}-\frac{a}{6 w} \frac{1}{\mathbf{k}^{4}}
$$

leads to the classical linear potential as

$$
V^{\mathrm{SIG}}(r)=\frac{M a}{24 \cdot \pi}\left[1+\frac{1}{8 w}\right] r,
$$

where one uses the relation [15]

$$
\int d^{3} \mathbf{k} \frac{e^{i \mathbf{k} \cdot \mathbf{r}}}{\mathbf{k}^{4}}=\frac{2 \pi}{r} r^{2} \int_{-\infty}^{\infty} d(k r) \frac{\sin (k r)}{(k r)^{3}}=-\pi^{2} r .
$$

This classical potential $V^{\mathrm{SIG}}(r)$ is 0 (finite) at the origin of $r=0$ and the latter part of this potential was found in Ref. [15]. Also, it is desirable to discuss the Newtonian potential for the conformal gravity. Yoon [16] criticized 
that Mannheim's conformal gravity [17] leads to negative linear potential, which is problematic from the point of view of fitting galaxy rotation curves. This requires positive linear potential. However, Mannheim [18] answered that Yoon had made an error in his analysis of the sign of linear coefficient. In this work, from the first term in (11), we read off positive linear potential when choosing positive $a$.

On the other hand, adding $2 R / \kappa^{2}$ to Eq.(3) leads to the FDG propagator [19]

$$
\mathcal{P}_{\mu \nu, \alpha \beta}^{\mathrm{FDG}}(k)=\left[\frac{1}{k^{2}}-\frac{1}{k^{2}-m_{2}^{2}}\right] P^{(2)}-\frac{1}{2}\left[\frac{1}{k^{2}}-\frac{1}{k^{2}-m_{0}^{2}}\right] P^{(0-s)}+(\cdots),
$$

where mass squared forms are given by

$$
m_{2}^{2}=\frac{2 a}{\kappa^{2}}, \quad m_{0}^{2}=-\frac{1}{\kappa^{2}} \frac{a}{w} .
$$

Here we require the non-tachyonic masses of $m_{2}^{2}>0$ and $m_{0}^{2}>0$ which determines the signs of $a$ and $w$ such that $a>0$ and $w<0$. This inequality implies the positivity of the coefficient in front of $r$ in $V^{\mathrm{SIG}}$ (11). Then, we obtain the famous classical potential

$$
V^{\mathrm{FDG}}(r)=-\frac{G M}{r}\left[1-\frac{4}{3} e^{-m_{2} r}+\frac{1}{3} e^{-m_{0} r}\right]
$$

when using

$$
V^{\mathrm{FDG}}(r)=\frac{\kappa_{4} M}{(2 \pi)^{3}} \int d^{3} \mathbf{k} e^{i \mathbf{k} \cdot \mathbf{r}} \mathcal{P}_{00,00}^{\mathrm{FDG}}(\mathbf{k})
$$

where

$$
\mathcal{P}_{00,00}^{\mathrm{FDG}}(\mathbf{k})=\frac{1}{2}\left[-\frac{1}{\mathbf{k}^{2}}+\frac{4}{3} \frac{1}{\mathbf{k}^{2}+m_{2}^{2}}-\frac{1}{3} \frac{1}{\mathbf{k}^{2}+m_{0}^{2}}\right] .
$$

Here we point out that in the limit of $r \rightarrow 0$, a massive ghost tensor contributes $4 / 3(=1+1 / 3)$ to the potential and a massive healthy scalar contributes $-1 / 3$ to the potential. The singularity cancellation occurs in the FDG. This model shows that the theory without any kind of nonlocality could be free from the Newtonian singularity. Interestingly, for $r \gg r_{0}$ where $r_{0}=$ $1 / \min \left(m_{2}, m_{0}\right)$, we get the usual Newtonian potential of $V^{\mathrm{FDG}} \approx-G M / r$. However, for $r \ll r_{0}$, one finds that

$$
\begin{aligned}
V^{\mathrm{FDG}} & \approx \frac{G M\left(m_{0}-4 m_{2}\right)}{3}+\frac{G M\left(4 m_{2}^{2}-m_{0}^{2}\right) r}{6}+\cdots \\
& =\frac{G M\left(m_{0}-4 m_{2}\right)}{3}+\frac{M a}{24 \cdot \pi}\left[1+\frac{1}{8 w}\right] r+\cdots .
\end{aligned}
$$


Here we observe that the last term is the same form as (11). Considering the condition of $a>0$ and $w<0$, the coefficient of $r$ is positive (negative) for $w<-0.125(w>-0.125)$. It is worth noting that the classical potential $V^{\text {SIG }}$ of the SIG is embedded as the linear term of the FDG when expanding $V^{\mathrm{FDG}}(r)$ around $r=0$. This may suggest a deep renormalization connection between SIG and FDG [2, 20].

\section{Renormalization of SIG}

We start by mentioning that the three coupling constants $a, w, c$ are dimensionless in (1). Considering a propagator behaving as $1 / k^{4}$, the SIG has been shown to be renormalizable and asymptotically free without including the Einstein-Hilbert term $2 R / \kappa^{2}$. Also, since the static potential (11) associated with $1 / k^{4}$-propagator is proportional to distance $r$, this would be a confining theory.

Before we proceed, we have to mention that the main purpose of [7] was the one-loop computation in the general theory including the Einstein-Hilbert and cosmological terms, and such a calculation was first performed in [20].

Let us remind that the effect of $c G B$ term is not relevant for one-loop renormalization, but it may play a role in renormalization group equations. However, the inclusion of $c G B$ made a little difference in some coefficients of the beta-functions [10, 13].

Hence, we consider the renormalization group equations in terms of two coupling constants $(a, w)$ which are relevant to determining the classical potential (11) 21]. In this case, the Weyl coupling a may be identified with the loop-expansion parameter, whereas the Ricci coupling $w$ will be seen to approach a UV fixed point. We renormalize the couplings $a$ and $w$ by introducing $\mu$ the renormalization parameter of dimensional regularization $(n=4-\epsilon)$. With $(4 \pi)^{2} t=\ln \left[\mu / \mu_{0}\right]$, the relevant beta-functions take the forms

$$
\begin{aligned}
\frac{d a}{d t} & =-\epsilon a-a^{2} \frac{\partial\left(a A_{1}\right)}{\partial a} \\
\frac{d w}{d t} & =a \frac{\partial\left[a\left(B_{1}-w A_{1}\right)\right]}{\partial a} .
\end{aligned}
$$

The one-loop divergences have provided the counterterms of $A_{1}$ and $B_{1}$ as $[9$, 13

$$
A_{1}=\frac{133}{10}, \quad B_{1}=\frac{10 w^{2}}{3}-5 w+\frac{5}{12} .
$$

Considering $a$ as a loop-expansion parameter with tree approximation of order $1 / a, A_{1}$ and $B_{1}$ imply that the one-loop divergences are independent 
of $a$. In the limit of $\epsilon \rightarrow 0(n \rightarrow 4)$, one finds the one-loop beta-functions

$$
\begin{aligned}
\frac{d a}{d t} & =-\frac{133}{10} a^{2} \\
\frac{d w}{d t} & =\left[\frac{5}{12}-w\left(5+\frac{133}{10}\right)+\frac{10}{3} w^{2}\right] a .
\end{aligned}
$$

The first equation (22) is a negative beta-function and thus, can be solved to be

$$
a(t)=\frac{a_{0}}{1+\frac{133}{10} a_{0} t},
$$

which shows that in the UV limit of $t \rightarrow \infty$, it manifests the asymptotic freedom for $a$. That is, the Weyl coupling $a$ is always asymptotically free from solely gravity self-interactions. For the Ricci coupling $w$, we find two roots of $0<w_{1}<w_{2}\left(w_{2}=5.47, w_{1}=0.023\right)$ from the beta-function (23). The coupling $w$ approaches the UV fixed point $w_{1}$ for $w$ within the UV attraction region $0<w<w_{2}$. A UV fixed point is located at $w=w_{1}$ and so, the coupling $w$ is also asymptotically free. Also, an IR fixed point is found at $w=w_{2}$. We note that the $w \rightarrow 0$ limit denotes a strong coupling limit where the one-loop analysis is not reliable [22. Hence, the action (3) is asymptotically free for $a>0$ and $w>0$ and thus, it is regarded as a UV complete theory.

\section{Discussions}

First of all, we have found the linear classical potential $V^{\mathrm{SIG}}(r)$ (11) from the SIG which is zero (finite) at the origin of $r=0$. The SIG provides another gravity, showing that the higher-derivative gravity without any kind of nonlocality could be free from the Newtonian singularity. This is because it had a massless tensor and scalar dipole propagator of $1 / k^{4}$. On the other hand, the SIG action (11) is renormalizable and asymptotically free for $a>0$ and $w>0$. The above statements show clearly that the SIG satisfies the conjecture which states that renormalzable higher-derivative gravity has a finite classical potential at the origin.

Furthermore, we observe that the inequality condition of $a>0$ and $w<$ -0.125 is compatible with the positivity of coefficient in front of $r$ in $V^{\mathrm{SIG}}$, implying that the SIG is a confining theory. In addition, it was known that the classical potential $V^{\mathrm{SIG}}$ is embedded as the linear term of the FDG when expanding $V^{\mathrm{FDG}}(r)$ around $r=0$. This may suggest a deep connection between SIG and FDG renormalizations. 
Initially, we have included the Gauss-Bonnet (GB) term to investigate its roles in both classical potential and one-loop renormalization in four dimensions. Taking into account the GB term $c G B$, it did not contribute to the graviton propagator on the Minkowski spacetimes and thus, to the classical potential. On the renormalization side, the effects of the Gauss-Bonnet term is not relevant for one-loop renormalization and renormalization group equation in four dimensions [9, 10]. This implies that the GB term which does not correspond to any graviton interaction in four dimensions, plays no crucial role in both classical potential and one-loop renormalization in four dimensions. That is, its role is trivial.

At this stage, we wish to discuss the unitarity issue of the SIG. As was shown in (5), the former propagator of $1 / k^{4}$ is considered as the degenerated limit of massless spin-2 pole and massive spin-2 ghost pole (massless tensor dipole) and the latter is the degenerated limit of massless spin- 0 pole and massive spin-0 ghost pole (massless scalar dipole). This implies that the SIG has ghosts. It was known that there are two possible ways to avoid ghosts as followed by FDG : nonlocal FDG [23, 24] or Lee-Wick gravity [25]. However, the SIG has no the Einstein-Hilbert term, implying that it gives rise to difficulty to remove the ghost-like states. Even though the SIG is renormalizable and asymptotically free, this theory is non-unitary.

Finally, the conformal gravity with $w=0$ in (1) provides the classical linear potential $V^{\mathrm{CG}}=[\mathrm{Ma} / 24 \pi] r$, while it is renormalizable without $R^{2}$ term [13]. For $a>0$, this gravity is a confining theory as well as a UV complete theory. Hence, the conformal gravity is regarded as the simplest model which satisfies the conjecture.

\section{Acknowledgement}

This work was supported by the National Research Foundation of Korea (NRF) grant funded by the Korea government (MOE) (No. NRF-2017R1A2B4002057). 


\section{References}

[1] L. Modesto, T. de Paula Netto and I. L. Shapiro, JHEP 1504, 098 (2015) doi:10.1007/JHEP04(2015)098 [arXiv:1412.0740 [hep-th]].

[2] K. S. Stelle, Phys. Rev. D 16, 953 (1977). doi:10.1103/PhysRevD.16.953

[3] B. L. Giacchini, Phys. Lett. B 766, $306 \quad$ (2017) doi:10.1016/j.physletb.2017.01.019 [arXiv:1609.05432 [hep-th]].

[4] A. Accioly, J. de Almeida, G. P. Brito and G. Correia, Phys. Rev. D 95, no. 8, 084007 (2017) doi:10.1103/PhysRevD.95.084007 arXiv:1702.07404 [hep-th]].

[5] A. Accioly, J. de Almeida, G. P. de Brito and W. Herdy, arXiv:1707.02083 [hep-th].

[6] D. G. Boulware, G. T. Horowitz and A. Strominger, Phys. Rev. Lett. 50, 1726 (1983). doi:10.1103/PhysRevLett.50.1726

[7] E. S. Fradkin and A. A. Tseytlin, Nucl. Phys. B 201, 469 (1982). doi:10.1016/0550-3213(82)90444-8

[8] F. David and A. Strominger, Phys. Lett. 143B, 125 (1984). doi:10.1016/0370-2693(84)90817-7

[9] I. G. Avramidi and A. O. Barvinsky, Phys. Lett. 159B, 269 (1985). doi:10.1016/0370-2693(85)90248-5

[10] G. de Berredo-Peixoto and I. L. Shapiro, Phys. Rev. D 71, 064005 (2005) doi:10.1103/PhysRevD.71.064005 hep-th/0412249].

[11] E. T. Tomboulis, Mod. Phys. Lett. A 30, no. 03n04, 1540005 (2015). doi:10.1142/S0217732315400052

[12] I. Antoniadis, P. O. Mazur and E. Mottola, Nucl. Phys. B 388, 627 (1992) doi:10.1016/0550-3213(92)90557-R hep-th/9205015.

[13] G. de Berredo-Peixoto and I. L. Shapiro, Phys. Rev. D 70, 044024 (2004) doi:10.1103/PhysRevD.70.044024 [hep-th/0307030].

[14] K. S. Stelle, Int. J. Mod. Phys. A 32, no. 09, 1741012 (2017). doi:10.1142/S0217751X17410123 
[15] L. Alvarez-Gaume, A. Kehagias, C. Kounnas, D. Lst and A. Riotto, Fortsch. Phys. 64, no. 2-3, 176 (2016) doi:10.1002/prop.201500100 arXiv:1505.07657 [hep-th]].

[16] Y. Yoon, Phys. Rev. D 88, no. 2, $027504 \quad$ (2013) doi:10.1103/PhysRevD.88.027504 [arXiv:1305.0163 [gr-qc]].

[17] P. D. Mannheim, Prog. Part. Nucl. Phys. 56, 340 (2006) doi:10.1016/j.ppnp.2005.08.001 astro-ph/0505266].

[18] P. D. Mannheim, Phys. Rev. D 93, no. 6, 068501 (2016) doi:10.1103/PhysRevD.93.068501 arXiv:1506.02479 [gr-qc]].

[19] Y. S. Myung, Phys. Rev. D 96, no. 6, 064026 (2017) doi:10.1103/PhysRevD.96.064026 [arXiv:1706.01173 [gr-qc]].

[20] J. Julve and M. Tonin, Nuovo Cim. B 46, 137 (1978). doi:10.1007/BF02748637

[21] M. B. Einhorn and D. R. T. Jones, Phys. Rev. D 91, no. 8, 084039 (2015) doi:10.1103/PhysRevD.91.084039 [arXiv:1412.5572 [hep-th]].

[22] B. Holdom and J. Ren, Phys. Rev. D 93, no. 12, 124030 (2016) doi:10.1103/PhysRevD.93.124030 arXiv:1512.05305 [hep-th]].

[23] L. Modesto, Phys. Rev. D 86, 044005 doi:10.1103/PhysRevD.86.044005 arXiv:1107.2403 [hep-th]].

[24] T. Biswas, E. Gerwick, T. Koivisto and A. Mazumdar, Phys. Rev. Lett. 108, 031101 (2012) doi:10.1103/PhysRevLett.108.031101 arXiv:1110.5249 [gr-qc]].

[25] L. Modesto and I. L. Shapiro, Phys. Lett. B 755, 279 (2016) doi:10.1016/j.physletb.2016.02.021 [arXiv:1512.07600 [hep-th]]. 